\title{
Análisis de la venta de entradas de un mega evento deportivo: el caso del Mundial de Natación
}

\author{
Análise da venta de entradas de um mega evento deportivo: o caso do Mundial de \\ Natación \\ Analysis of ticket sales for a mega sporting event: the case of the Swimming World Cup
}

Francesc Solanellas Donato ${ }^{\mathrm{a}, \mathrm{b}, \mathrm{c}}$ (D), Joshua Muñoz Vázquez ${ }^{\mathrm{a}, \mathrm{b}, c *}$ (D)

Palabras clave:

Venta de entradas;

Eventos deportivos; Marketing deportivo; Comportamiento del consumidor.

\section{Keywords:}

Ticketing;

Sporting events;

Sport marketing;

Consumer behavior.

\section{Palavras-chave:}

Venda de entradas;

Eventos esportivos; Marketing esportivo; Comportamento do consumidor.

\begin{abstract}
Resumen
En el presente artículo se muestra una fotografía del resultado del proceso de venta de entradas del Mundial de Natación, así como se analiza el comportamiento del consumidor en el conjunto de este proceso. Se analizaron descriptivamente 46.181 transacciones para las variables de precio, asistencia, capacidad, disciplina deportiva, horario de competición, momento y canal de compra. Además, se desarrolló un modelo de regresión polinómica que explicó el 48,51\% de la variabilidad de entradas vendidas. Gracias a la implementación de una política dinámica de precios, se logró una asistencia del $66,8 \%$ con un $53,1 \%$ de ventas anticipadas. De media, se compraron 4,22 entradas en cada transacción y una mayor asistencia se relacionó con un mayor volumen de ingresos. Los resultados pueden ser considerados para aplicar medidas en la planificación de la propia competición, así como para la orientación de estrategias comerciales.
\end{abstract}

\begin{abstract}
This article shows a photograph of the result of the ticket sales process for the Swimming World Cup, as well as an analysis of consumer behavior throughout this process. 46,181 transactions were descriptively analyzed for the variables of price, attendance, capacity, sports discipline, competition schedule, moment, and purchase channel. In addition, a polynomial regression model was developed that explained $48.51 \%$ of the variability of tickets sold. Thanks to the implementation of a dynamic pricing policy, attendance of $66.8 \%$ was achieved with $53.1 \%$ of anticipated sales. On average, it was bought 4.22 tickets in each transaction, and higher attendance was associated with higher revenue volume. The results can be considered to apply measures in the planning of the competition itself, as well as for the orientation of commercial strategies.
\end{abstract}

\section{Resumo}

Este artigo apresenta uma fotografia do resultado do processo de venda de ingressos da Copa do Mundo de Natação, bem como uma análise do comportamento do consumidor ao longo desse processo. 46.181 transações foram analisadas descritivamente para as variáveis de preço, assiduidade, lotação, disciplina esportiva, cronograma de competição, horário e canal de compra. Além disso, foi desenvolvido um modelo de regressão polinomial que explicou 48,41\% da variabilidade dos ingressos vendidos. Graças à implementação de uma política de preços dinâmica, foi alcançado um atendimento de 66,8\% com 53,1\% das vendas antecipadas. Em média, foram adquiridos 4,22 ingressos em cada transação, e o maior comparecimento foi associado ao maior volume de receita. Os resultados podem ser considerados para aplicação de medidas no panejamento da própria competição, bem como para a orientação de estratégias comerciais.

\footnotetext{
anstituto Nacional de Educación Física de Cataluña (INEFC), Universidad de Barcelona (UB). Barcelona, España. bDepartamento de Gestión y Ocio del Deporte (INEFC). Barcelona, España. 'Grupo de Investigación Social y Educativa de la Actividad Física y el Deporte (GISEAFE). Barcelona, España.
}

\footnotetext{
*Autor correspondiente:

Joshua Muñoz Vázquez

E-mail: joshuamunozv@gmail.com
} 


\section{INTRODUCCIÓN}

Desde el punto de vista de la gestión de un evento deportivo, la venta de entradas es decisiva para el éxito general. Por un lado, la venta de entradas proporciona una fuente importante de ingresos (Stewart, 2014) y, por otro lado, aseguran la asistencia de público al evento. De hecho, una asistencia superior a un evento deportivo se ha relacionado con una mayor obtención de ingresos de un determinado equipo o instalación (Howard y Crompton, 2014). Además, conviene subrayar que, en los eventos deportivos los espectadores juegan un papel fundamental ya que co-crean calidez junto con los atletas (Chappelet, 2013). En consecuencia, y dado que las entradas no pueden ser vendidas una vez el evento ha terminado, es imperativo que las organizaciones deportivas maximicen constantemente las oportunidades de venta, sin importar el entorno en el que operan (Popp et al., 2017).

Dada la relación directa entre el comportamiento de compra de un consumidor y las decisiones en las políticas de precios de los organizadores, se cree que una comprensión profunda del proceso de toma de decisiones del consumidor deportivo ayudará a las organizaciones deportivas a implementar estrategias de precios y de gestión de ingresos más efectivas. De esta manera, y debido a que una de las formas más obvias de predecir el comportamiento futuro es revisar el comportamiento pasado, contextualizarlo y extrapolarlo al futuro, el propósito de esta investigación es el de proporcionar una fotografía del resultado final del proceso de venta de entradas del Mundial de Natación de Barcelona. Contribuyendo de esta manera a la literatura sobre la venta de entradas en los mega-eventos deportivos.

\section{REVISIÓN DE LITERATURA}

Hoy en día, con varias opciones para comprar, los consumidores deportivos se ven obligados a especular antes de actuar (Dwyer et al., 2013). De ahí que, adaptar los precios a los cambios en las condiciones circundantes durante cortos períodos de tiempo, se considere un factor crítico para administrar con éxito un negocio en la industria de servicios (Kretsch, 1995).

A lo largo de los años, las organizaciones deportivas han desarrollado estrategias de precios avanzadas con el fin de maximizar los ingresos. Shapiro y Drayer (2014) relataron como, desde el año 2000, el mercado de la venta de entradas a eventos deportivos ha experimentado diferentes transformaciones. A principios del siglo XXI los equipos profesionales comenzaron a experimentar con precios variables según los diferentes tipos de partidos y adversarios, día de la semana, temporada, etc. A partir de esta segmentación inicial de precios, las estrategias siguieron evolucionando hacia precios dinámicos en función de la demanda.

En este sentido, "[...] comprender la respuesta de los consumidores a los precios y los cambios de precios es de vital importancia" (Drayer et al., 2012, p. 192), entre otras cosas, para satisfacer continuamente las necesidades y deseos únicos de los clientes potenciales (Dwyer et al., 2018). Precisamente, en el modelo sobre el comportamiento del consumidor que Sheth y Mittal (2003) desarrollaron, se diferenciaban tres etapas que en cierta manera destacan los conceptos abordados en la literatura científica sobre la venta de entradas en eventos deportivo: (1) comprender al cliente; (2) comprender el comportamiento del cliente; y (3) crear valores de mercado para el cliente.

Drayer et al. (2012), señalaron como los estudios anteriores que examinaron las variaciones en la demanda de los consumidores de un evento deportivo, habían utilizado una variedad de medidas a través de las que se podría comprender el comportamiento del cliente, incluidas la ventaja de jugar en casa (Boyd y Boyd, 1998) la incertidumbre de los resultados (Falter y Perignon, 2000; Forrest y Simmons, 2002), o, variables tradicionales relacionadas con el juego, como el rendimiento del equipo y el jugador (Drayer et al., 2012; Drayer y Shapiro, 2009; Lemke et al., 2010), así como variables basadas en las estrategias de ventas que las organizaciones implementaban, como el precio de las entradas (Rishe y Mondello, 2003, 2004), la disponibilidad de entradas (Shapiro y Drayer, 2012), o el tiempo en avanzado de compra (Dwyer et al., 2013; Moe et al., 2011).

En suma, Borland y MacDonald (2003) mostraban en su recopilación de estudios que no hay escasez de investigaciones sobre los factores que influyen en la demanda del consumidor. Sin embargo, la gran mayoría de estudios no exploraron las percepciones de los consumidores sobre estas influencias y/o su disposición a pagar las entradas. Vacío que recientemente autores como Drayer y Shapiro (2011), Kaiser et al. (2019) y Kemper y Breuer (2016) han intentado cubrir en sus investigaciones sobre la disposición a pagar que los diferentes segmentos de espectadores pueden tener.

En definitiva, estos estudios, que ayudan a entender la respuesta de los consumidores y su comportamiento de compra de entradas durante un evento deportivo, contribuyen en la práctica para ayudar a las organizaciones en la implementación de estrategias en el proceso de venta de entradas, así como a la selección de mercados objetivo-adecuados, pues se trata de un aspecto primordial para desarrollar programas de marketing exitosos (Dubois y Duquesne, 1993).

El Campeonato Mundial de Natación podría verse como un evento único en comparación con otras competiciones que se gestionan semanalmente. Además, este campeonato es como organizar cinco grandes eventos en uno: natación, natación sincronizada, saltos, waterpolo y, por primera vez en un Mundial de Natación, saltos de gran altura.

En este caso, la propiedad del evento corresponde a la FINA (Federación Internacional de Natación) que delegó la organización en el Comité Organizador. La venta 
de entradas es una tarea enorme y la forma en que un comité organizador de un evento a gran escala maneja el problema tiene un impacto significativo en los eventos, y la relación de este con la comunidad (Thamnopoulos y Gargalianos, 2002). En el evento deportivo objeto de análisis de la presente investigación, los principales canales de ventas de entradas fueron de forma online (durante todo el proceso de venta de entradas) y a través de las taquillas físicas (tan solo las 3 semanas de competición).

Por consiguiente, como se adelantaba en la introducción, con el propósito de proporcionar una instantánea del desenlace del proceso de venta de entradas, el problema o pregunta de investigación que se aborda es el siguiente: ¿cuál fue el resultado del proceso de venta de entradas del Mundial de Natación? Se trata de una pregunta de tipo descriptivo (Heinemann, 2008), a través de la que se pretende detallar el desenlace del proceso de venta de entradas del objeto de estudio. Además, llegados a este punto, es preciso volver a destacar el modelo de Sheth y Mittal (2003) sobre el comportamiento del consumidor. Pues, aportar datos sobre la segunda etapa del modelo propuesto por los autores (comprender el comportamiento del cliente) será un objetivo indirecto de esta investigación.

\section{MÉTODO}

\section{CONTEXTUALIZACIÓN DEL EVENTO}

El Mundial de Natación de 2013, objeto de estudio, se desarrolló en 4 instalaciones diferentes: Arena Sant Jordi, la Piscina Municipal de Saltos, la Piscina Bernat Picornell y el Puerto de Barcelona. La capacidad total de ventas de las diferentes instalaciones deportivas se muestra en la Tabla 1.

Es destacable que la ceremonia de clausura no se ha considerado necesario incorporarla en la Tabla 1 ya que fue un evento abierto al público en general y se desarrolló en un espacio abierto dentro del recinto del Anillo Olímpico de Montjuic.

\section{DESCRIPCIÓN DE VARIABLES}

Para el estudio descriptivo se tuvieron en cuenta variables como el precio de las entradas, la asistencia, la capacidad de las instalaciones, las disciplinas deportivas, el horario de la competición, el momento y el canal de compra de las entradas. Variables que también fueron contempladas por el Comité Organizador a la hora de controlar el flujo de la venta de entradas.

\section{RECOGIDA Y ANÁLISIS DE DATOS}

Los datos analizados son todas las transacciones de ventas realizadas durante los 8 meses previos al Campeonato de Natación de Barcelona y durante la propia competición. Se analizaron un total de 46.181 transacciones: el $78,3 \%$ del total de 59.000 . Esto es debido a que se filtraron aquellas transacciones que correspondían a un valor de transacción 0 , como, por ejemplo, las entradas gratuitas para patrocinadores o compromisos institucionales.

Para permitir la manipulación adecuada de los datos, se procedió a la limpieza de la base de datos y se trataron estadísticamente con el programa SPSS.

Se extrajeron las frecuencias, y se calcularon en términos absolutos y proporcionalmente. Además, con respecto a la evolución de las ventas por semana, se desarrolló un modelo de regresión polinómica para examinar el impacto de estos factores en el precio de las entradas.

Con el análisis de regresión polinómica se pretendió proponer un modelo explicativo que pudiera predecir el número de entradas vendidas al final del evento. La regresión polinómica es un tipo de análisis de la regresión en donde la relación entre la variable independiente " $x$ " $y$ la variable dependiente " $y$ " es modelada como un polinomio de grado " $n$ " en " $x$ ". Este es el motivo por el cual se considera un caso concreto de regresión lineal múltiple. Este tipo de regresión dibuja una curvatura al modelo, introduciendo nuevos predictores que se consiguen al elevar algunas variables originales a distintas potencias.

Tabla 1. Capacidad para cada disciplina.

\begin{tabular}{cccc}
\hline & Capacidad & Sesiones & Capacidad Total \\
\hline Ceremonia de apertura & 7.375 & 1 & 7.375 \\
Natación & 7.836 & 16 & 125.376 \\
Saltos & 3.400 & 16 & 54.400 \\
Saltos de gran altura & 500 & 3 & 1.500 \\
Natación sincronizada & 7.836 & 10 & 78.360 \\
Waterpolo masculino & 2.500 & 14 & 35.000 \\
Waterpolo femenino & 2.500 & 14 & 35.000 \\
Natación en aguas abiertas & 500 & 1 & 500 \\
Total & $\mathbf{2 6 . 7 1 1}$ & $\mathbf{7 5}$ & $\mathbf{2 8 0 . 1 5 1}$ \\
\hline
\end{tabular}

Fuente: Elaboración propia. 
Por tanto, el objetivo de un modelo de regresión es tratar de explicar la relación que existe entre una variable dependiente (variable respuesta) y un conjunto de variables independientes. El número de entradas era por tanto la variable dependiente " $y$ " y la variable independiente " $x$ " el número de semanas que quedaban para el evento.

\section{RESULTADOS}

En las Figuras 1 y 2 se analizan en profundidad varias variables (capacidad, asistencia, ingresos totales, precio promedio y disciplina deportiva). Por una parte (Figura 1), se pueden ver las variables cruzadas entre el $\%$ de ingresos por disciplina, respecto el \% de asistencia

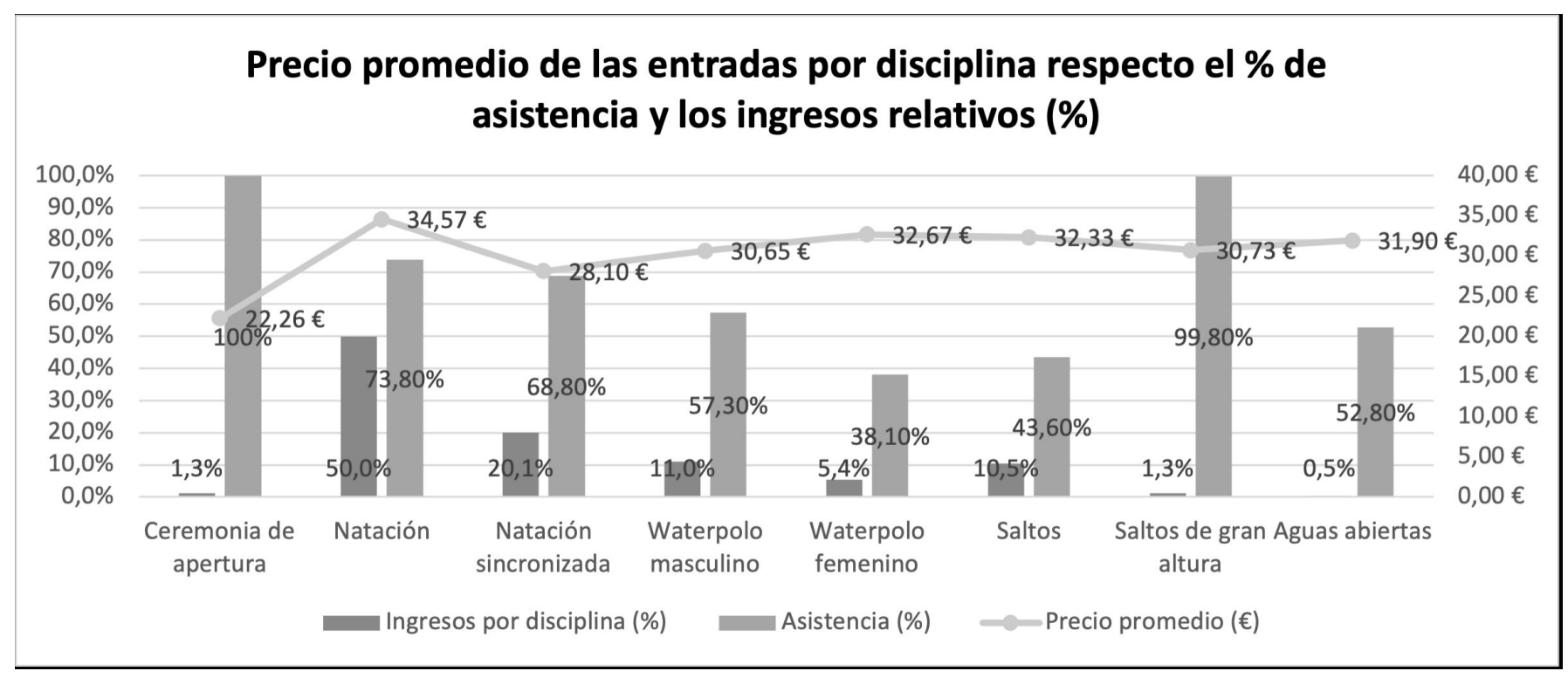

Figura 1. Precio promedio de la entrada según disciplina respecto el \% de asistencia de la capacidad y los ingresos por disciplina (\%).

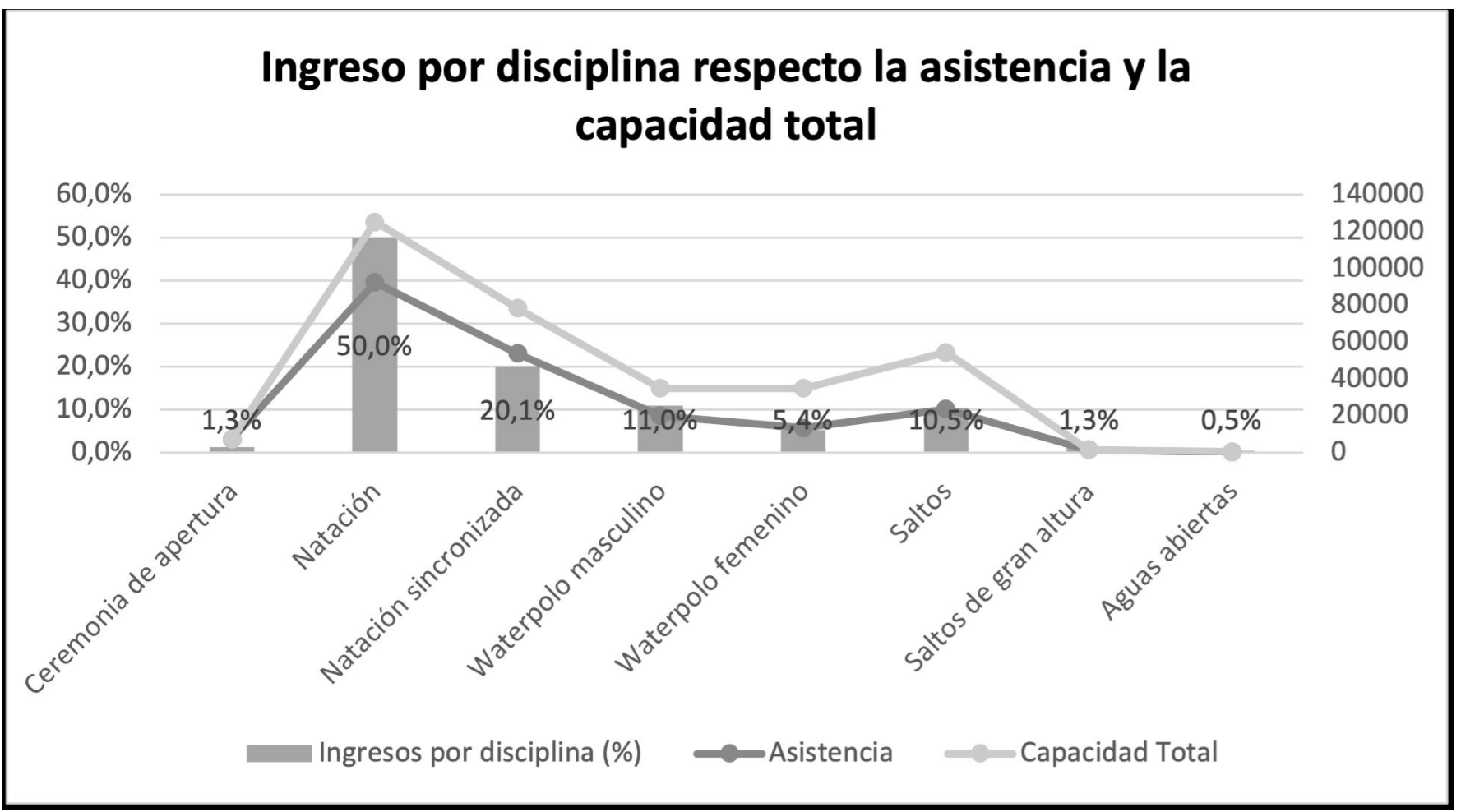

Figura 2. Ingreso por disciplina respecto la asistencia y la capacidad total. 
a los eventos y el precio promedio de la entrada para cada disciplina; y por otra, en la Figura 2, las variables del precio promedio de las entradas en función de la capacidad total y de la asistencia a los eventos.

La asistencia, medida como porcentaje de la capacidad total de una instalación, es probablemente el primer resultado para tener en cuenta de un megaevento deportivo. En el caso que nos ocupa, como se desprende de la Figura 1, la asistencia promedio general fue del $62,03 \%$, siendo la ceremonia de apertura el único evento con aforo completo y el waterpolo femenino el evento con menor asistencia: $38,1 \%$ sobre el total de la capacidad.

También se muestran los resultados de facturación de cada uno de los eventos respecto el total de las cifras informadas. La natación fue la disciplina que supuso un mayor porcentaje $(50,00 \%)$ de las ventas totales, seguida de la natación sincronizada $(20,1 \%)$, el waterpolo masculino $(11,01 \%)$ y los saltos $(10,5 \%)$.

En el caso que nos ocupa, se comprueba como el precio promedio de las entradas en función de la disciplina, fueron de las más económicas (ceremonia de apertura-22,26€) a las más caras (natación-34,57€).

Por otra parte, se comprueba que una mayor asistencia se asocia a un mayor volumen de ingresos (Figura 2). Sin embargo, disciplinas como los eventos de la ceremonia de apertura y saltos de gran altura fueron eventos en los que se consiguió una asistencia muy alta respecto al total de la capacidad, pero que los ingresos representaron porcentajes muy bajos respecto el total de los ingresos. En el resto de los eventos, con capacidad de asistencia similar (natación y natación sincronizada con mayores capacidades al resto), se comprueba que, como el precio promedio por entrada fue parecido entre las disciplinas $(S D=3,78 €)$, la asistencia a los eventos fue la variable que marcaría la representatividad en el \% de ingresos totales.
Siguiendo la estrategia comercial de venta de entradas del evento, se puede observar (Figura 3) que, en términos generales, se decidió iniciar las ventas con precios más altos y, progresivamente, conforme se acercaba el día de la competición, se procedió a aplicar diferentes políticas de descuentos.

Cabe destacar la diferencia entre el precio promedio total de la transacción $(87,41 €)$ y el precio promedio de la entrada $(33,84 €)$. Este último se calcula de la división del total de transacciones por número de entradas compradas. Los consumidores compraron 4,22 entradas en promedio con una desviación estándar de 11,46 entradas. El máximo alcanzó la cifra de 100.

La Figura 3 también muestra la evolución de las ventas durante todo el período. Se puede apreciar que durante los meses anteriores las ventas fueron bastante constantes, mientras que en los días de competición las ventas aumentaron considerablemente. Se puede ver como apenas un día antes del inicio de la competición se alcanzó el $53,1 \%$ de las ventas totales.

Desde un punto de vista de los datos absolutos, el modelo de regresión expuesto en la Figura 4 explica que el $48,51 \%$ de la variabilidad en las entradas vendidas se debe a la semana de compra. Sin embargo, si examinamos al detalle la comparativa entre las disciplinas acuáticas, se puede ver como aparentemente cada una tiene modelos diferentes. Por ejemplo, la natación fue la disciplina con mayores ventas; y también la que tuvo la fecha de venta promedio más avanzada. Por otro lado, el waterpolo femenino fue la disciplina que menos vendió y tuvo un promedio de ventas más cercano a la fecha de la competición.

Si se analiza el comportamiento de los compradores en función de los días de la semana en los que realizaron las compras, se puede ver (Figura 5) que no hay grandes diferencias en porcentaje, pero sí en la tendencia durante la semana. Se podría decir que los porcentajes más altos

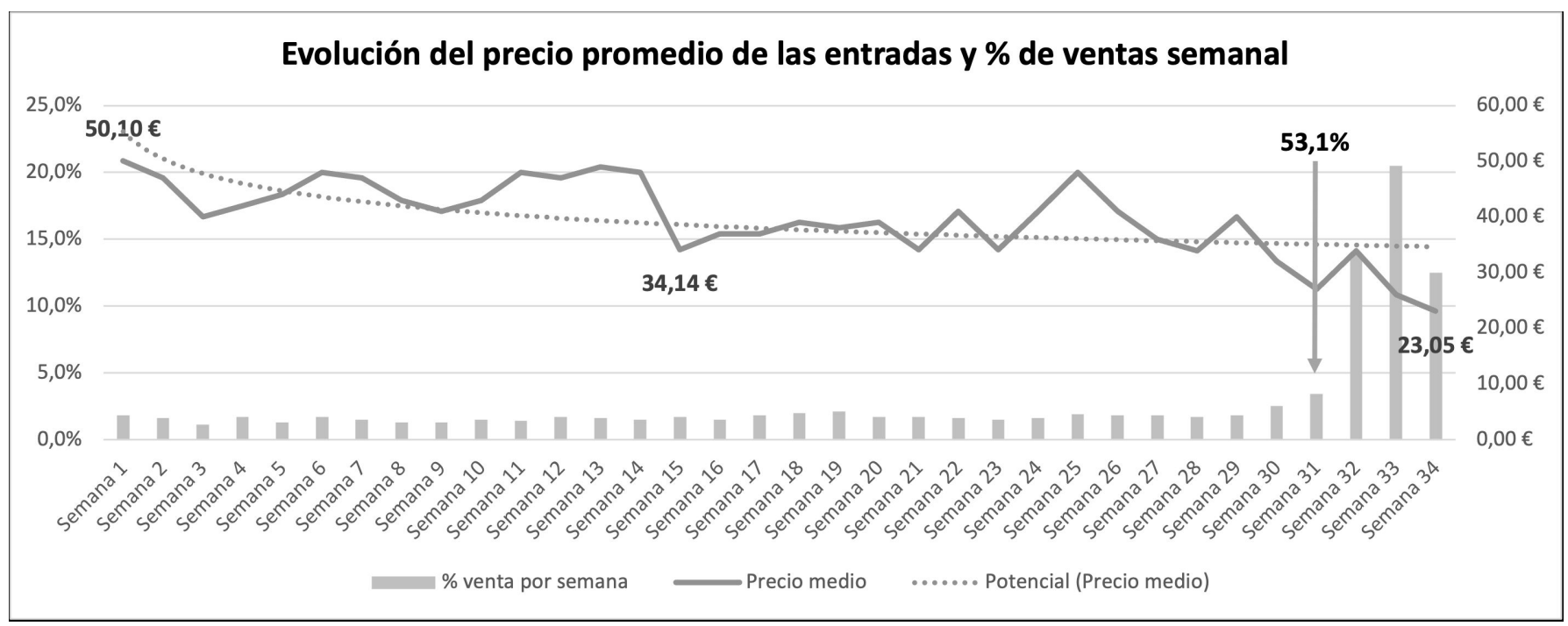

Figura 3. Evolución del precio promedio de las entradas y \% de ventas semanal. 


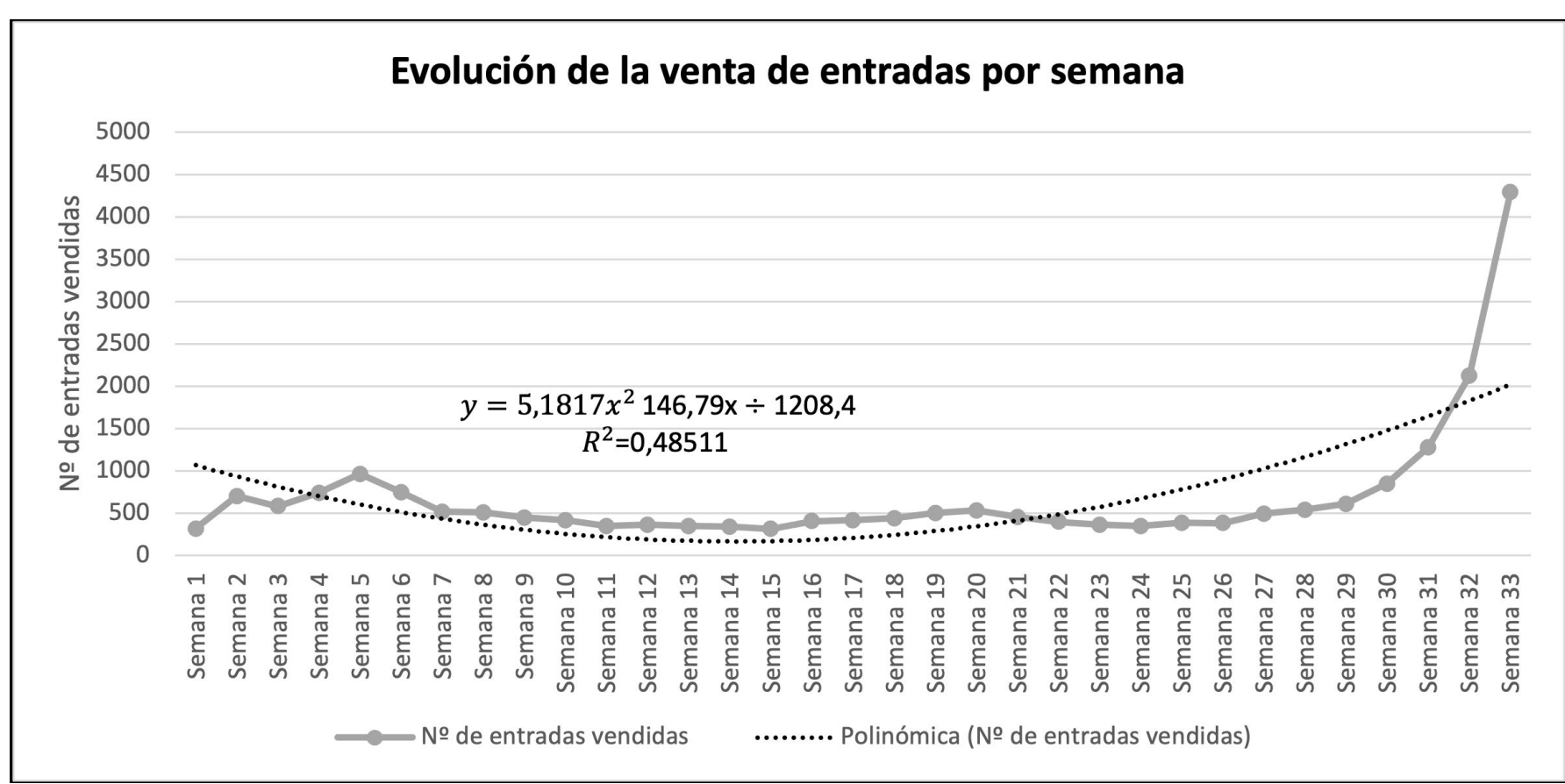

Figura 4. Evolución de las ventas por semana.

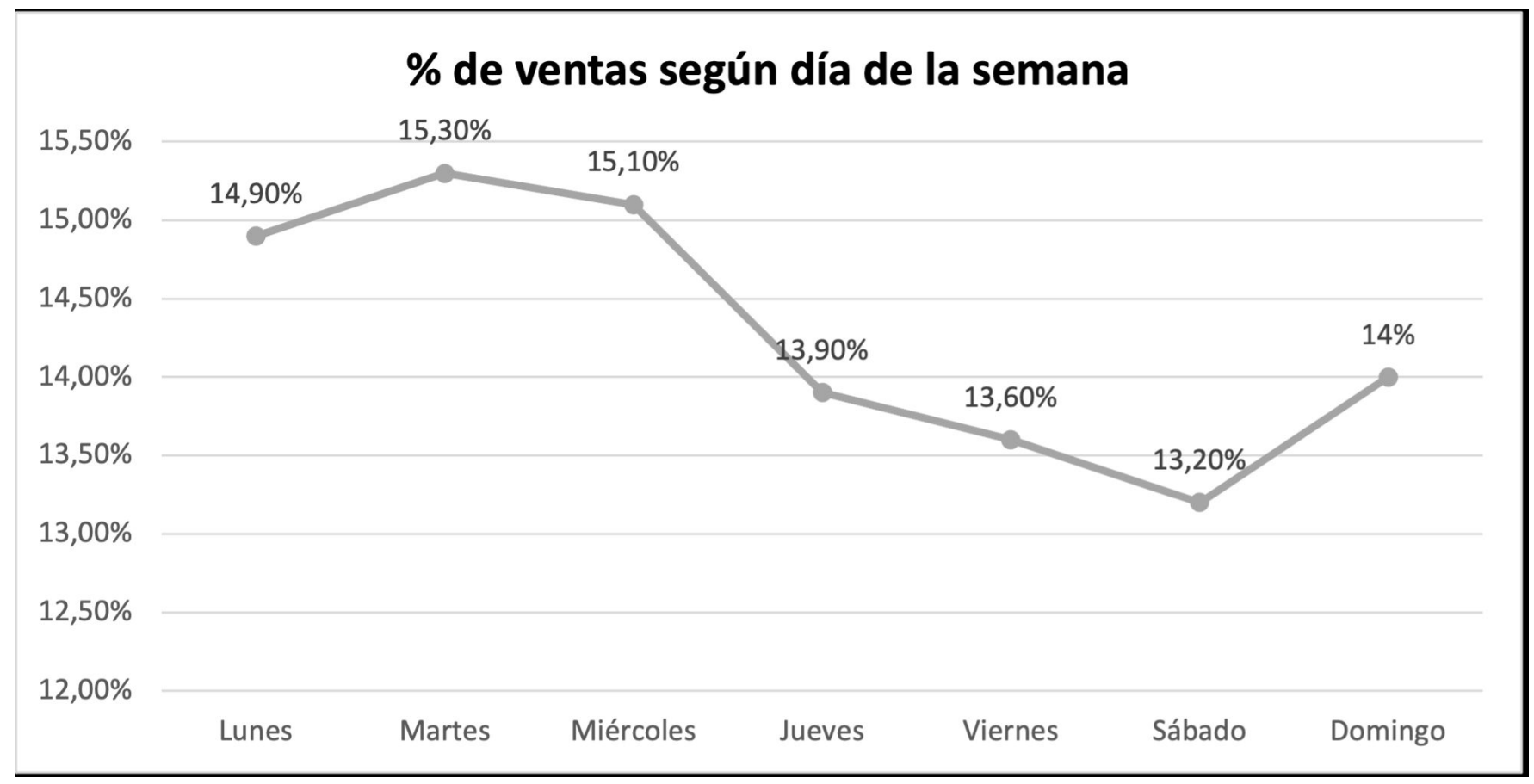

Figura 5. Porcentaje de boletos comprados en diferentes días.

(>14\%) se encuentran entre el domingo y el miércoles. De jueves a sábado la tendencia desciende y a partir del domingo los porcentajes vuelven a subir.

Otro elemento clave a la hora de entender el comportamiento de los espectadores del Campeonato del Mundo de Natación, es cómo hacer coincidir el calendario de las sesiones con el volumen de transacciones de esas sesiones. Se puede observar en la Figura 6 que la mayoría de las sesiones se planificaron durante las tardes de los días laborables (37,50\%), las tardes de los fines de semana (25\%) y las mañanas de los días laborables (24\%). Sin embargo, el número de transacciones se concentró principalmente para las sesiones de tarde de fin de semana, con un $43,20 \%$. 
Asimismo, otro indicador importante a la hora de analizar el comportamiento de los consumidores es el porcentaje de ventas realizadas a través de la web o a través de las taquillas instaladas en las últimas tres semanas, que corresponden a las semanas de competición. Como se puede observar en la Figura 7, el $84,83 \%$ de todas las ventas se realizaron a través del sitio web. El 15,17\% restante correspondió a ventas en las taquillas.

\section{DISCUSIÓN}

Tras el análisis de los datos, se puede comprobar como durante el Mundial de Natación analizado se consiguió, en cierta manera, lo que autores como Howard y Crompton (2014) entendían que debe de ser el objetivo principal en la gestión de venta de entradas: maximizar los ingresos sin poner en riesgo la asistencia de los espectadores.

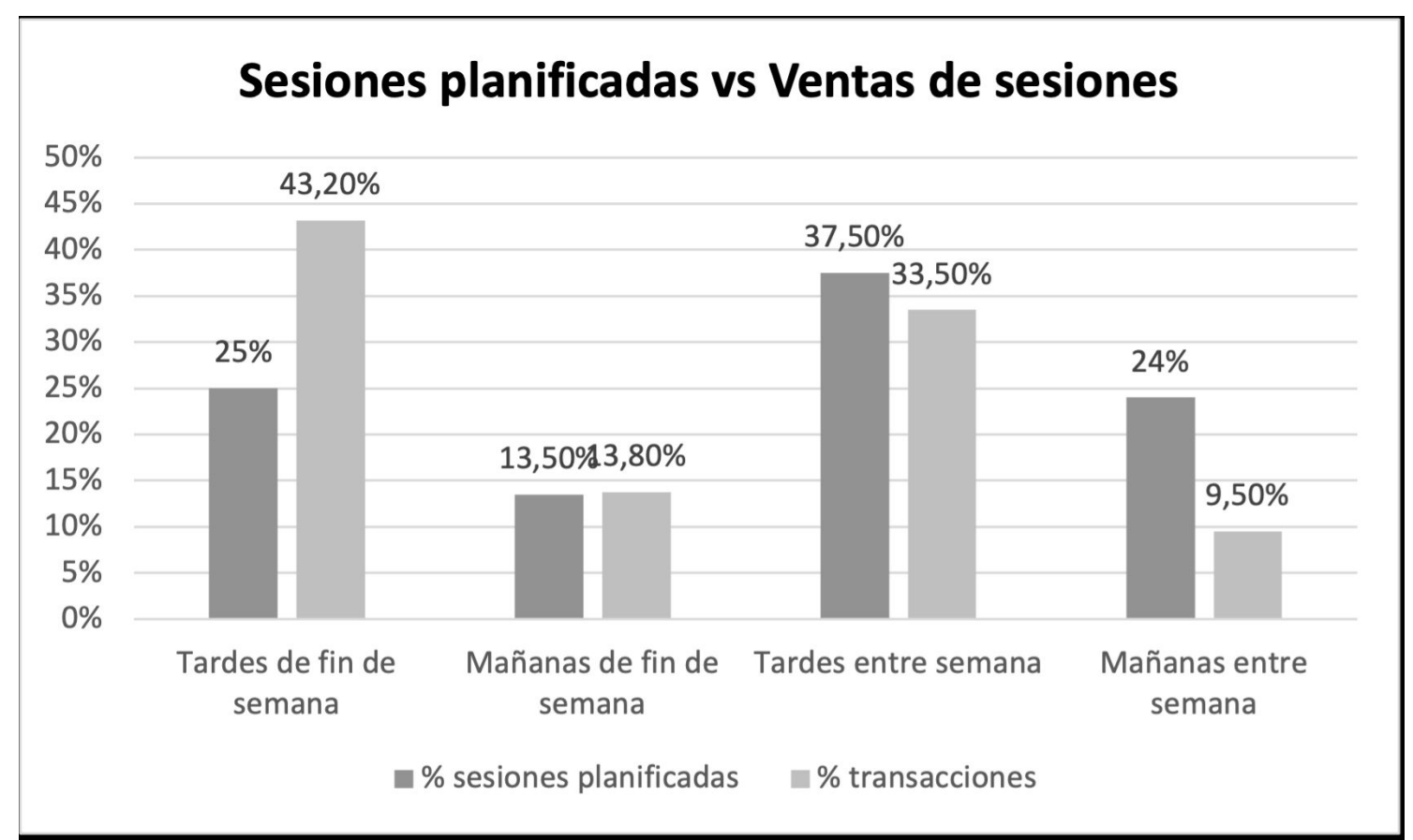

Figura 6. Sesiones planificadas versus ventas de sesiones.

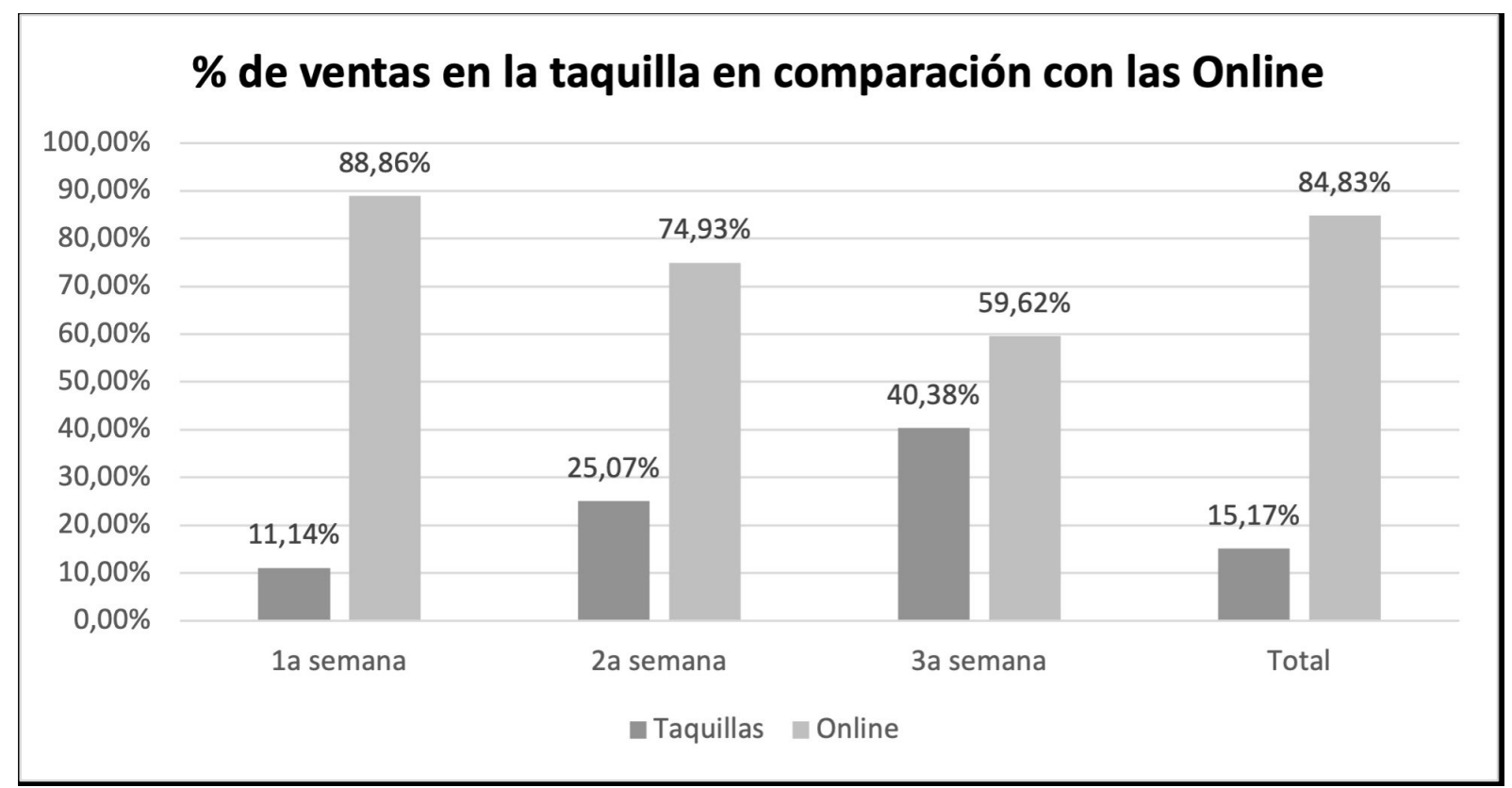

Figura 7. \% de ventas en la taquilla en comparación con las ventas Online. 
Asimismo, a partir de los resultados, se pueden explicar algunos aspectos que nos permiten comprender la respuesta de los consumidores a los precios y los cambios de estos. Algo que Drayer et al. (2012) entendían de vital importancia para la gestión de la venta de entradas.

Como se ha podido ver en la evolución del precio promedio de las entradas (Figura 3 ), los responsables de la venta de entradas adaptaron los precios en el tiempo en función de diversas condiciones. En efecto, se implementó una estrategia dinámica de precios que según Drayer et al. (2012) puede considerarse como una estrategia de precios adecuada para eventos deportivos. Se observa como se decidió iniciar las ventas con precios más altos, posiblemente para atraer a las personas más interesadas, o que tuvieran que planificar sus viajes con anticipación, y progresivamente, para atraer más público en general se prosiguió con descuentos en los precios de las entradas. En este sentido, se podría especular que, con el objetivo de maximizar los ingresos, la organización buscase llegar con rangos de precios más altos a los consumidores deportivos que tienen una identificación más fuerte con el equipo. En su estudio, Drayer y Shapiro (2011) determinaron que los fanáticos que tienen una identificación o lealtad más fuerte con el equipo están dispuestos a pagar más para ver jugar al equipo. Si más no, los resultados del proceso de venta de entradas analizado concuerdan con los hallazgos de estudios como el de Dwyer et al. (2013) y Moe et al. (2011) donde comprobaron que los precios disminuyeron a medida que se acercaba el evento. Sin embargo, en la literatura sobre estrategias dinámicas de precios se puede encontrar como el precio variable de las entradas se ha manifestado bajo diferentes enfoques. Entre ellos, como el llevado a cabo por el Comité Organizador del evento analizado, aquel que reconoce el concepto de ciclo vital, en el que, con el tiempo, el valor del producto es probable que disminuya (Ennis, 2020). En la otra cara de la moneda, también se encuentran estrategias que operan de la manera opuesta, fijando los precios en valores más altos conforme se acerca la competición y gratificando así la compra anticipada de entradas. Si más no, para implementar una correcta estrategia dinámica de precios, que tenga por objetivos estimular la demanda y maximizar los ingresos, requerirá de una alta capacidad de adquisición y análisis de datos, pues se trata de algo imprescindible para tomar decisiones sobre los precios (Ennis, 2020). En este sentido, como anticipaba Abeza (2020) y Ennis (2020), el Big Data podría jugar un papel fundamental en un futuro próximo. Proporcionando información invaluable sobre los consumidores y las reacciones de estos a los cambios de precios, ayudando así a las organizaciones en la toma de decisiones con la posibilidad de implementar el análisis de datos en tiempo real.

En la Figura 3 se muestra como, con la estrategia implementada en el proceso de venta de entradas, se consiguió un $53,1 \%$ de ventas anticipadas previas al inicio del evento. Dato que podría considerarse como relevante pues según autores como Dwyer et al. (2013) o Hendrickson (2012) la venta anticipada de entradas proporciona a una organización deportiva la seguridad de unos ingresos garantizados, así como la posibilidad de anticiparse para poder disponer de los recursos necesarios para brindar el servicio necesario. Sin embargo, examinando al detalle la comparativa entre las disciplinas acuáticas, veríamos como cada una tiene modelos diferentes. Por ejemplo, la natación fue la disciplina con mayores ventas; y también la que tuvo la fecha de venta promedio más avanzada. Por otro lado, el waterpolo femenino fue la disciplina que menos vendió y tuvo un promedio de ventas más cercano a la fecha de la competición. En este sentido, respecto a la venta de entradas de las últimas 3 semanas (iniciado el campeonato), cabe destacar que algunos de los factores que más podrían influir serian tales como los resultados deportivos, el tipo de competición, etc. Por ejemplo, siendo España el país donde se organizaba el evento deportivo, el equipo nacional de Waterpolo Femenino se alzó con la medalla de oro, hecho que pudo contribuir a una mayor asistencia por parte del público local. De facto, variables como el rendimiento del equipo y el jugador (Drayer et al., 2012; Drayer y Shapiro, 2009; Lemke et al., 2010) o la ventaja de jugar en casa (Boyd y Boyd, 1998) han sido identificadas por diversos autores como factores que influyen positivamente en la venta de entradas.

Como se deduce de la Figura 2, y pese a que, en términos generales, los resultados concuerdan con otros estudios en los que se identifica que una mayor asistencia de público se relaciona con un mayor volumen de ingresos (Howard y Crompton, 2014), se puede identificar un cierto margen de mejora en la política de precios y descuentos implementada para cada disciplina. Por ejemplo, mientras que los precios promedio más bajos pudieron ayudar a una mayor asistencia en disciplinas como la natación sincronizada, precios un poco más altos en disciplinas como waterpolo femenino y saltos pudieron ser un limitante o un obstáculo para asegurar una mayor asistencia a los eventos. Sin embargo, desde el punto de vista de maximizar los ingresos, en el caso de los saltos, pese a que la asistencia estuvo por debajo del $50 \%$ del total de la capacidad, los ingresos representaron un 10,5\% (Figura 1) respecto el total de los ingresos del evento, porcentaje muy parecido al de otras disciplinas como Waterpolo Masculino (11\%) que tuvieron una mayor asistencia respecto a la capacidad total. A pesar de esto, es difícil establecer una relación causa-efecto en la asistencia a los eventos deportivos, ya que esto dependerá de diversos factores como el entorno, la cultura deportiva del país, de los resultados deportivos, etc. (Hansen y Gauthier, 1989; Paul y Weinbach, 2013).

Para comprender el comportamiento del cliente, así como la demanda de los consumidores, es importante destacar como los consumidores compran las entradas. Así, se pudo observar que los consumidores compraron 
un promedio de 4,22 entradas en cada transacción realizada con una desviación estándar de 11,46, algo que indicaría que los espectadores acudieron al evento en grupo. De hecho, las transacciones con un mayor volumen de compra de entradas alcanzaron la cifra de 100 entradas, lo que significa que algunas de las entradas se compraron en packs.

Por otra parte, la capacidad total de una instalación deportiva es uno de los aspectos importantes a considerar a la hora de establecer la política de precios de las entradas para un evento deportivo, puesto que esta marcará también la disponibilidad de entradas y la percepción que los consumidores puedan tener sobre esta. En concreto, en el evento objeto de estudio de esta investigación, se puede comprobar (Figura 1) como en prácticamente todas las disciplinas se consiguieron porcentajes de asistencia por encima del $50 \%$ de la capacidad máxima de las instalaciones, no siendo esta un limitante (excepto para la ceremonia de apertura -100\%y los saltos de gran altura $-99,8 \%-)$. Sin embargo, se considera que puede haber un cierto margen de mejora, sobre todo en aquellas disciplinas que consiguieron porcentajes de asistencia bajos respecto a la capacidad total (waterpolo femenino -38,1\%-y saltos -43,6\%-).

Si se analiza el comportamiento de los compradores en función de los días de la semana (Figura 5), se comprueba como los días con mayores transacciones fueron martes y miércoles. Comportamiento difícil de explicar en esta investigación pero que sin embargo se trata de datos que creemos importantes mostrar y tener en cuenta para que las organizaciones puedan implementar sistemas de gestión de ingresos con el objetivo de "[...] determinar precios de acuerdo con los niveles de demanda previstos" (Kimes et al., 1998, p. 33). Por otra parte, entendiendo la dinámica intrínseca del evento deportivo analizado, y con los resultados expuestos en la (Figura 6), creemos que concentrar las competiciones por las tardes (tanto entre semana, como en fin de semana) podría asegurar una mayor asistencia a los eventos, $y$, en consecuencia, una mayor venta de entradas.

Respecto al canal de compra (Figura 7) se pudo comprobar como pese a que la venta de entradas en las taquillas cobra más importancia conforme se acerca el evento, la venta a través de canales online representó el $84,83 \%$ del total de ventas durante las últimas 3 semanas del evento. En las semanas previas, el $100 \%$ de las transacciones se dieron online ya que los espectadores no disponían de la opción de compra a través de las taquillas físicas. La venta online, puede ser una herramienta que contribuya a la maximización de los ingresos, ya que internet permite a los clientes buscar fácilmente el producto de su elección dentro de su rango de precios deseado, al mismo tiempo que permite a las organizaciones experimentar con diversas estrategias de precios y establecer varios segmentos de mercado (Kung et al., 2002).

\section{CONCLUSIONES}

La venta de entradas supone un gran esfuerzo para la entidad organizadora del evento y la forma en que un comité organizador de grandes eventos lo gestiona tiene un impacto significativo (Thamnopoulos y Gargalianos, 2002). Los resultados de esta investigación son únicos por el tamaño y la calidad de la muestra. De hecho, con los datos totales para el evento, se podría pensar más en términos de población total que en términos de muestra. Es, a su vez, un claro ejemplo de lo que podría ser el análisis del proceso de venta de entradas en eventos deportivos para futuros estudios longitudinales que vayan relacionados con el Big Data.

Establecer las motivaciones, el perfil y el tipo de compra de los consumidores es y será determinante para orientar las estrategias comerciales. Creemos que nuestro estudio es buena prueba de ello, para un megaevento en el que se determinó una tasa de participación del $66,8 \%$, se ha podido revelar el modelo de venta implementado, teniendo en cuenta variables como el precio de las entradas, la capacidad de las instalaciones, el horario de la competición y el momento y canal de compra de las entradas. Describiendo así, de una manera exhaustiva, el proceso de venta de entradas del Mundial de Natación de Barcelona y aportando datos sobre el comportamiento de los consumidores.

El análisis aquí mostrado tiene varias implicaciones prácticas. En primer lugar, de manera directa, se podrían estudiar posibles medidas a aplicar en la planificación de la propia competición (Mundiales de Natación), referidas a las disciplinas deportivas y sus horarios, así como de orientar estrategias y promociones comerciales de manera más efectiva que permitieran mejorar los resultados de las organizaciones deportivas en cuanto a los ingresos y la asistencia de público al evento se refiere. Por otro lado, creemos que la propuesta de análisis expuesta en el presente artículo, con las diferentes variables identificadas y sus posibles relaciones, puede servir como modelo para el análisis del proceso de venta de entradas de otros mega-eventos deportivos.

Como se ha visto hasta ahora, la venta de entradas se ha convertido en un tema habitual en la literatura sobre gestión deportiva. Sin embargo, todavía podemos considerarlo como un tema a desarrollar en un futuro próximo ya que no es tan evidente obtener grandes bases de datos o información de deportes profesionales o mega-eventos. Es aquí donde creemos que nuestra investigación contribuye a la literatura específica que hay hasta la fecha, pues gracias a la posibilidad de disponer de la base de datos del Mundial de Natación se ha podido analizar la totalidad de las transacciones que se realizaron durante el proceso de venta de entradas.

\section{LIMITACIONES E INVESTIGACIONES FUTURAS}

Sin duda, el estudio tiene limitaciones. En primer lugar, porque la información presentada no llega a aportar 
datos más precisos sobre los propios consumidores en cuanto a su perfil: género, edad, consumidor o no, zona geográfica, experiencias previas, etc. Sin lugar a duda, es algo a tener en cuenta en futuros estudios. Además, se necesitan nuevas investigaciones para comprender cómo los consumidores pueden dividirse en segmentos más pequeños según sus hábitos de compra (Shapiro y Drayer, 2012) y tampoco fue objeto de estudio la disposición de los fans a pagar y las variables que influyen en dicha disposición, ni la venta de entradas en un entorno de mercado secundario al oficial.

Creemos que para obtener más información de los espectadores hubiese sido interesante incluir un breve cuestionario a los compradores con el objetivo de poder profundizar en el análisis, y así establecer una segmentación del público que compró entradas, ya que esto ayudaría a la selección de mercados objetivoadecuados y a desarrollar programas de marketing exitosos (Dubois y Duquesne, 1993). Aspecto que podría ser considerado a implementar en investigaciones futuras, pero que muestra algunas deficiencias debido al riesgo potencial de hacer una experiencia de compra más larga y tediosa con la posibilidad de reducir el número total de ventas.

Asimismo, el trabajo con Big Data podría jugar un papel fundamental a la hora de "[...] personalizar soluciones para los fanáticos en base a algo más que una corazonada, basada en los datos" (Abeza, 2020, p. 668), personalización que luego se traduce en componentes transaccionales.

Por último, como se trata de una investigación con carácter ideográfico, se debe considerar que algunos resultados no puedan generalizarse. De esta manera, pese a que el evento analizado se trata del Mundial de Natación que logró una de las mayores asistencias de todos los Mundiales de Natación organizados hasta la fecha, en futuras investigaciones, con la posibilidad de obtener los datos de ediciones anteriores, se podría considerar la comparativa en cuanto a las variables aquí estudiadas.

\section{IMPLICACIONES TEÓRICAS Y PRÁCTICAS}

Aunque se trate de un estudio de casos, los resultados pueden ser relevantes para todas las organizaciones deportivas ya sean de carácter local o internacional. De esta manera, la investigación contribuye a comprender el proceso de la venta de entradas en un mega evento deportivo y mantiene abierto el debate sobre las posibilidades futuras en un área que se encuentra en constante evolución tanto para académicos, como para gestores. Así pues, esperamos que los resultados de la investigación ayuden a concienciar a los gestores deportivos de las complejidades que implica el proceso de venta de entradas y comprenderlas de la mejor manera posible para maximizar los beneficios de las entradas.

\section{FINANCIAMIENTO}

No existe apoyo económico alguno para la realización de la investigación.

\section{CONFLICTOS DE INTERÉS}

Los autores declaran que no existe ningún conflicto de intereses.

\section{REFERENCIAS}

Abeza G. Big data in sport industry: interview with Michal Lorenc, head of industry: ticketing and live events at Google. Int J Sport Commun. 2020;13(4):663-9. http:// dx.doi.org/10.1123/ijsc.2020-0272.

Borland J, MacDonald R. Demand for sport. Oxf Rev Econ Policy. 2003;19(4):478-502. http://dx.doi.org/10.1093/ oxrep/19.4.478.

Boyd DW, Boyd LA. The home field advantage: implications for the pricing of tickets to professional team sporting events. J Econ Finance. 1998;22(2-3):169-79. http://dx.doi. org/10.1007/BF02771486.

Chappelet JL. Spectating the games. In: Girginov V, editor. Handbook of the London 2012 Olympic and Paralympic Games. New York: Routledge; 2013. Chapter 15. http:// dx.doi.org/10.4324/9780203126486.ch10.

Drayer J, Shapiro SL, Lee S. Dynamic ticket pricing in sport: a conceptual approach. Sport Mark Q. 2012;21:184-94.

Drayer J, Shapiro SL. An examination into the factors that influence consumers' perceptions of value. Sport Manage Rev. 2011;14(4):389-98. http://dx.doi.org/10.1016/j. smr.2010.11.001.

Drayer J, Shapiro SL. Value Determination in the Secondary Ticket Market: A Quantitive Analysis of the NFL Playoffs. Sport Marketing Quarterly. 2009, 18(1): 5-13.

Dubois B, Duquesne P. The market for luxury goods: Income versus culture. Eur J Mark. 1993;27(1):35-44. http://dx.doi. org/10.1108/03090569310024530.

Dwyer B, Drayer J, Shapiro SL. Proceed to checkout? The impact of time in advanced ticket purchase decisions. Sport Mark Q. 2013;22(3):166-80.

Dwyer B, Lecrom C, Greenhalgh G. Exploring and measuring spectator sport fanaticism. Communication and Sport. 2018;6(1):58-85. http://dx.doi. org/10.1177/2167479516679411.

Ennis S. Sports marketing. a global approach to theory and practice. Switzerland: Springer; 2020. http://dx.doi. org/10.1007/978-3-030-53740-1.

Falter JM, Perignon C. Demand for football and intramatch winning probability: an essay on the glorious uncertainty of sports. Appl Econ. 2000;32(13):1757-65. http://dx.doi. org/10.1080/000368400421101.

Forrest D, Simmons R. Outcome uncertainty and attendance demand in sport: the case of English soccer. J R Stat Soc Ser A Stat Soc. 2002;51(2):229-41.

Hansen $H$, Gauthier R. Factors affecting attendance at professional sport events. J Sport Manage. 1989;3(1):1532. http://dx.doi.org/10.1123/jsm.3.1.15. 
Heinemann K. Introducción a la metodología de la investigación empírica: en las ciencias del deporte. Barcelona: Editorial Paidotribo; 2008.

Hendrickson $\mathrm{H}$. View from the field: business intelligence in the sports world. Sport Mark Q. 2012;21:136-7.

Howard DR, Crompton JL. Financing sport. 3rd ed. Morgantown, WV: Fitness Information Technology; 2014.

Kaiser M, Ströbel T, Woratschek H, Durchholz C. How well do you know your spectators? A study on spectator segmentation based on preference analysis and willingness to pay for tickets. Eur Sport Manag Q. 2019;19(2):178-200. http://dx.doi.org/10.1080/16184742.2018.1499790.

Kemper C, Breuer C. How efficient is dynamic pricing for sport events? Designing a dynamic pricing model for Bayern Munich. Int J Sport Finance. 2016;11(1):4-25.

Kimes SE, Chase RB, Choi S, Lee PY, Ngonzi EN. Restaurant revenue management: applying yield management to the restaurant industry. Cornell Hotel Restaur Adm Q. 1998;39(3):32-9. http://dx.doi.org/10.1177/001088049803900308.

Kretsch SS. Airline fare management and policy. In: Jenkins $D$, editor. Handbook of airline economics. New York: McGraw-Hill; 1995.

Kung M, Monroe KB, Cox JL. Pricing on the Internet. J Prod Brand Manage. 2002;11(5):274-88. http://dx.doi. org/10.1108/10610420210442201.

Lemke RJ, Leonard M, Tlhokwane K. Estimating attendance at Major League Baseball games for the 2007 season. J Sports Econ. 2010;11(3):316-48. http://dx.doi. org/10.1177/1527002509337212.

Moe WW, Fader PS, Kahn B. Buying tickets: capturing the dynamic factors that drive consumer purchase decision for sporting events. In: MIT Sloan Sports Analytics Conference; 2011; Boston. Proceedings. Boston: SSAC; 2011.
Paul RJ, Weinbach AP. Determinants of dynamic pricing premiums in Major League Baseball. Sport Mark Q. 2013;22:152-65.

Popp N, Simmons J, McEvoy CD. Sport ticket sales training: perceived effectiveness and impact on ticket sales results. Sport Mark Q. 2017;26(2):99-109.

Rishe PJ, Mondello MJ. Ticket price determination in professional sports: an empirical analysis of the NBA, NFL, NHL, and Major League Baseball. Sport Mark Q. 2004;13(2):104-12.

Rishe PJ, Mondello MJ. Ticket price determination in the National Football League: a quantitative approach. Sport Mark Q. 2003;12(2):72-9.

$<$ jrn>Shapiro SL, Drayer J. A new age of demand-based pricing: An examination of dynamic ticket pricing and secondary market prices in Major League Baseball. Journal of Sport Management. 2012; 26(6): 532-546. https://doi. org/10.1123/jsm.26.6.532.</jrn>

Shapiro SL, Drayer J. An examination of dynamic ticket pricing and secondary market price determinants in Major League Baseball. Sport Manage Rev. 2014;17(2):145-59. http:// dx.doi.org/10.1016/j.smr.2013.05.002.

Sheth J, Mittal B. Customer behavior: a managerial perspective. 2nd ed. Cincinnati: South-Western College Pub.; 2003.

Stewart B. Sport funding and finance. 2nd ed. London: Routledge; 2014.

Thamnopoulos Y, Gargalianos D. Ticketing of large-scale events: the case of Sydney 2000 Olympic Games. Facilities. 2002;20(1/2):22-33. http://dx.doi. org/10.1108/02632770210414263. 